NASA Technical Memorandum 103946

\title{
Multidisciplinary Computational Aerosciences
}


NASA Technical Memorandum 103946

\section{Multidisciplinary Computational Aerosciences}

Paul Kutler, Ames Research Center, Moffett Field, California

June 1992

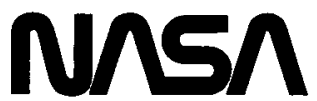

National Aeronautics and

Space Administration 


\section{Summary}

As the challenges of single disciplinary computational physics are met, such as computational fluid dynamics, computational structural mechanics, computational propulsion, computational aeroacoustics, computational electromagnetics, etc., scientists have begun investigating the combination of these single disciplines into what is being called multidisciplinary computational aerosciences (MCAS). The combination of several disciplines not only offers simulation realism but also formidable computational challenges. The solution of such problems will require computers orders of magnitude larger than those currently available. Such computer power can only be supplied by massively parallel machines because of the current speed-of-light limitation of conventional serial systems. Even with such machines, MCAS problems will require hundreds of hours for their solution. To efficiently utilize such a machine, research is required in three areas that include parallel architectures, systems software and applications software. The main emphasis of this paper is the applications software element. Examples that demonstrate application software for multidisciplinary problems currently being solved at NASA Ames Research Center are presented. Pacing items for MCAS are discussed such as solution methodology, physical modeling, computer power and multidisciplinary validation experiments.

\section{Introduction}

The use of computational fluid dynamics (CFD) in the aerospace business has steadily increased during the past decade and especially during the last several years. The primary reasons for this usage are the enhanced, validated applications software, available computer power to run that software and the past successful application of CFD to "real world" design problems. According to Holst et al. (ref. 1), computer hardware execution speed has increased by a factor of about 15 over the past decade and by over 200 during the past two decades. It can safely be said that the increased use of computational simulations has improved the efficiency of aerospace vehicle performance while at the same time reducing their cost to design.

The design of advance aerospace vehicles requires improvements in such properties as thrust, weight, and stability that is pushing the state of the art in these technologies. As a result, development costs using conventional technology are becoming prohibitively expensive. To reduce these costs, more emphasis is being placed on computer simulations by the aerospace designers and developers to provide data normally obtained from expensive wind tunnel or large-scale testing. If numerical simulations are to begin supplementing such testing, they must include the complex physical processes that exist on actual aerospace vehicles. This requires modeling physical processes such as propulsion/airframe interaction, flutter, surge, engine unstart and flameout.

The discipline of computational fluid dynamics has proven its value to many in the aerospace profession (for its simulation requirements, Boeing currently uses CFD $30 \%$ of the time versus $70 \%$ for experimentation; its projected that in the near future, the use of CFD will grow to $60 \%$ ), and it is routinely being used to simulate flows about vehicles and components that in some instances don't yet fully represent "real" aerospace configurations. The overarching goal for computational fluid dynamics or more appropriately "multidisciplinary computational aerosciences" (MCAS) in the aerospace field is to simulate the actual flow field about a computer optimized, realistic aerospace vehicle at true flight conditions in a reasonable amount of time on the computer. Phenomena such as shock and expansion waves, vortices, shear layers, separation, reattachment and unsteadiness are part of the actual flow field that must be predicted. A realistic configuration consists of a structure that is geometrically accurate, flexes under aerodynamic loads, has deflecting control surfaces that perturb the flow field, possesses propulsion systems that interact with the airframe and radiates acoustic, electromagnetic and infrared signatures. True flight conditions consist of the actual speed, attitude and altitude, all of which can vary with time. A reasonable amount of time on the computer depends on the need for the data. Use for design studies requires a quick turnaround time, probably on the order of one hour. Use for design validation, that might supplement or compliment an experiment or flight test, would not require such a short time.

Modeling the flow about realistic aerospace vehicles will require computing capabilities that currently do not exist. Computer performance today is limited by circuit speeds and thus the speed of light. The advent of computers based on parallel architectures offers the computational scientist an opportunity to solve his problem in a reasonable amount of time and thus should have an impact on multidisciplinary problems. Pacing items for computational aerosciences problems thus include computer hardware, systems software and applications software mapped efficiently onto the computer.

The simulation of an actual aircraft is truly multidisciplinary in nature and offers numerous challenges to the CFD scientist. The dimensions of the computational challenge are depicted graphically in figure 1. Displayed on the vertical axis is the equation set complexity, displayed on the horizontal axis is the geometric complexity and displayed on the inclined axis are the 
disciplinary interactions. In the early stages of development of the CFD discipline, activity was focused at the origin of this graph, that is, simple gas-dynamic equation sets were being applied to simple geometries. As the discipline matured, more descriptive equation sets were being solved for more complex geometries. However, these were still only being applied to predict the aerodynamics. Similarly, other single computational disciplines were advancing in an analogous fashion such as structures, acoustics, propulsion and controls. Computational fluid dynamicists today are capable of solving the Reynolds-averaged, Navier-Stokes equations about complete aircraft to predict the viscous flow field. They are also capable of solving that same equation set coupled with a simple structural model for wings. The discipline is thus heading towards multidisciplinary numerical simulation.

To that end, NASA is currently involved in a national research program entitled High-Performance Computing and Communication (HPCCP). One part of the NASA element involves the solution of computational aeroscience grand challenge problems, that is, multiple disciplinary problems, on massively parallel computers. The primary objective of the computational aeroscience applications portion of HPCCP is to develop robust computational methods and the associated pilot application computer programs that will enable integrated multidisciplinary analysis and design of advanced aerospace systems on massively parallel computers.

This program is unique in that it will require teams of scientists working together with expertise in computer science, computational science and numerous physical disciplinary sciences. One goal of the program is to make available computational power 1000 times greater than that existing today to solve the computational grand challenge problems. Research for this element of the program is focusing on the development of algorithms for multidisciplinary equation set coupling, algorithm/ architecture mapping of the multidisciplinary models, multidisciplinary optimization techniques, artificial intelligence procedures for multidisciplinary simulation and analysis, and application of these technologies to airframes, propulsion systems, and vehicle systems.

Challenges offered the CFD scientist include coupling the equations governing the necessary disciplines, developing the appropriate algorithms to solve that all inclusive set of equations and efficiently programming those procedures on the computer. Solution of such a set of equations is estimated to require a teraFLOPS $\left(10^{12}\right)$ computer and between 10 and $100 \mathrm{hr}$ per solution. Examples of MCAS are discussed in the next section.

\section{Multidisciplinary Examples}

Several examples are discussed below that demonstrate the MCAS program being pursued at the NASA Ames Research Center. Typical examples include highperformance aircraft, high-speed civil transport configurations and hypersonic vehicles. These problems were selected because of the following reasons: (1) development of technology for the numerical simulation of these vehicles will help the U.S. aerospace industry, (2) their simulation involves strongly-coupled multidisciplinary phenomena, and (3) their solution, it is believed, is tractable for advanced parallel computers. In each example discussed below, the plans for the project under NASA's HPCCP are first discussed followed by the current status and latest results of the project.

\section{High-Performance Aircraft .}

Multidisciplinary numerical simulations about highperformance aircraft are planned under HPCCP that include the disciplines of aerodynamics, engine stability, and aircraft controls (ref. 1). This is one of two major elements under that program. These disciplines will be integrated for two different but related problems; a powered lift aircraft undergoing a transition maneuver from hover to forward flight and a multi-role fighter undergoing a low-speed, high-g turn. Both of these simulations involve unsteady maneuvers and as such will require advanced computational resources, i.e., massively parallel computers. The propulsion challenges involved in this element are concentrating on simulating the behavior of the engine operating with distorted flow at its inlet. In addition to these computations, others are planned involving hover in ground effect and transonic cruise. A classical aerodynamic control system with a jet-implemented reaction control system will be employed for the poweredlift transition simulation while a classical aerodynamic control system with advanced forebody devices and thrust vector control system will be used for the multi-role fighter simulation. Following validation, it is expected that a necessary and accurate design and analysis tool for powered-lift aircraft will result.

Progress to date on this problem has yielded a capability to model the flow field surrounding the YAV-8B Harrier in low-velocity, jet-borne flight (ref. 2). Ground effect flow fields about powered-lift aircraft, such as the Harrier, are highly complex. Because of scaling effects, testing of these types of flows in small wind tunnels does not always produce an accurate representation of what would happen in flight. Thus, full-scaled powered wind tunnel tests are required to quantify the ground effects on vehicle performance. Powered-lift aircraft operating in ground 
effect can expect hazards such as hot-gas ingestion, foreign object damage and the "suckdown" effect. Therefore, numerical simulation offers an accurate cost effective technique for predicting the performance of poweredlift aircraft near the ground. Incorporated in the simulation is an engine component deck which models the response of an engine, given the throttle position and inlet temperature and pressure; it returns values for the mass flow and nozzle exit temperature.

A typical simulation for the flow field about the Harrier is shown in figure 2 . The flow conditions consist of 30 knots forward speed, angle of attack $=8.0^{\circ}$, at $30 \mathrm{ft}$ above the ground with the exhaust nozzles rotated $81^{\circ}$ below horizontal. There were 2.8 million grid points used in the calculation. The chimera overset grid method was utilized to break the flow field and 18 blocks were employed. This simulation solves the Reynolds-averaged, Navier-Stokes equations. A diagonalized, approximately factored solution algorithm was used to solve the governing equations. The computation was performed on a Cray YMP 8/128 using 40 single processor CPU hours and running at a rate of 130 MFLOPS (million floating point operations per second). Run-time storage requirements were $128 \mathrm{MB}$ (million bytes) of main memory and $740 \mathrm{MB}$ of secondary memory.

\section{High-Speed Civil Transport}

One of the two major projects under HPCCP deals with the High-Speed Civil Transport (HSCT). In this effort, the disciplines of aerodynamics, structural dynamics, combustion chemistry and controls will be integrated in a series of computational simulations about a supersonic cruise commercial aircraft. Focused research efforts will occur in three areas: (1) the ability to accurately and efficiently simulate landing conditions and transonic to supersonic cruise of a transport aircraft on advanced parallel computers, (2) the efficient coupling of the aerodynamic, propulsion, structures, and control disciplines, and (3) the efficient implementation of multidisciplinary design and optimization on advanced parallel computers.

The HSCT performance flight profile is constrained by three points that determine its design. These include supersonic cruise, transonic flight and landing. For supersonic cruise, airframe/propulsion integration and optimization is the major concern. For this multidisciplinary effort an aerodynamic analysis code, propulsion code and structural model for static deformations will be coupled together. These will be linked with an optimization code to search the design space for a configuration with an improvement in performance over the baseline design.
Aeroelasticity is a major concern for the HSCT in the transonic regime. The coupled fluid/structures code will be used to predict flutter boundaries for the HSCT. By adding an automatic control system for flutter suppression, this phenomena can be suppressed. Numerical simulations will be performed that model the flow about the HSCT in a landing configuration, that is, at large angle of attack and with flaps deployed. In addition, the tip vortex evolution and longevity during landing will be computed using a vortex tracking procedure.

All of these large-scale computations will be ported to parallel machines after successfully porting the singlediscipline versions of the individual codes. Correlations will be made with serial-processor calculations and experimental data made available from NASA's HighSpeed Research Program.

Computations to date on configurations in support of NASA's High-Speed Research Program include wing optimization using an Euler analysis, wing-body-nacelle for a low-boom configuration using the parabolized Navier-Stokes (UPS) equations and a wing-body configuration using the PNS equations on the Intel parallel machine.

Figure 3 shows the results computed, using the NASA Ames ARC3D code (S. Ryan, private communication), on the Intel iPSC/860 for the flow about an HSCT wing-body geometry. The flow conditions were $\operatorname{Minf}=2.1$ and angle of attack $=4.75^{\circ}$; the grid contained 450,250 points for this inviscid single block solution. Using 32 processors, the solution converged in 900 iterations, requiring $3.3 \mathrm{hr}$, or approximately $29 \mathrm{msec}$ per point per iteration. Using all 128 processors, execution speed increased to $11 \mathrm{msec}$ per point per iteration, which compares favorably with the performance of $13 \mathrm{msec}$ per point per iteration for the same code running on a single processor of a Cray YMP. As can be seen the results compare favorably to the solution generated by the UPS code on the Cray YMP.

\section{Hypersonic Vehicle}

Another multidisciplinary problem that NASA Ames. is undertaking under the HPCCP and its hypersonic program involves numerical simulation about very high-speed flight NASP (National Aero-Space Plane) derived vehicles (NDV's) (ref. 1). These are hypersonic, airbreathing aerospace vehicles. Key disciplines involved in this simulation are aerothermodynamics, structures, propulsion, and controls. The effort is focused on simulating the internal and external flow for this vehicle and coupling aerothermodynamics, finite-rate air and combustion chemistry, structures and controls on massively parallel computers. To accurately model the external flow for the 
high-speed portion of the flight about these vehicles will require a finite-rate air chemistry model (ref. 3). In addition, to simulate the flow in the combustion module will require a hydrogen-air combustion model. Important elements of this effort are the structural and thermal loads on these vehicles.

At NASA Ames, work is continuing on the development of applications software for simulating the flow about hypersonic vehicles. Computational fluid dynamics is being used to supplement limited experimental testing capabilities in the hypersonic regime. Continuous flow tunnels are non-existent for the flow conditions of interest, or those that do exist require too much power to operate. In addition, shock tunnels and ballistic ranges have short test durations and yield limited information.

To predict the hypersonic flow about an aerospace vehicle requires a mathematical model that is accurate enough to yield forces and moments, heat transfer, inlet mass capture and kinetic energy efficiency. The governing equations are the Reynolds-averaged Navier-Stokes in either the thin-layer or parabolized form. For hypersonic flow in which air chemistry is important, models for equilibrium air, nonequilibrium air and frozen flow are employed. Two classes of schemes are being utilized to solve the governing equations. They are flux-split schemes and Riemann solvers. Coupling of the airchemistry or species continuity equations and Reynoldsaveraged Navier-Stokes equations is required to solve hypersonic problems. Two approaches for coupling the equation sets are termed direct and loose. The direct approach requires, for an implicit procedure, the inversion of a large matrix while the loosely coupled approach requires solving the Navier-Stokes and air-chemistry equations separately. The former approach requires considerably more computer time than the latter approach.

For code validation purposes the McDonnell-Douglas Generic Option, Blended Wing-Body configuration has been extensively used. It possesses many of the features that future hypersonic vehicles will have including a small nose radius, compression ramps on the forebody, and an afterbody expansion surface. Experiments have been performed on this model in shock tunnels up to Mach 19. Because of the low total temperature of the flow, real-gas effects were not present in the experiment. A numerical simulation, however, was performed (ref. 4) at flow conditions that do generate nonequilibrium effects.

The results of that calculation are shown in figure 4 . This figure shows the computed pressure contours in various flow-field cross sections for a generic hypersonic vehicle. The solution was computed using the upwind parabolized Navier-Stokes solver UPS with flow conditions chosen to simulate Mach 16 flight at an altitude of approximately
$120,000 \mathrm{ft}$. Finite-rate (nonequilibrium) air chemistry was modeled using a loosely coupled species continuity solver. Atomic oxygen contours, plotted here in red, indicate that dissociation occurs primarily behind the strong shock near the blunt nose. Trace amounts of dissociation also result from the high temperatures generated within the boundary layer. The dissociated gas generated at the nose is swept toward the lower centerline as a natural result of the lower surface geometry and gradually recombines. Evident in the pressure contours are the bow shock wave and embedded shocks resulting from two lower surface compression ramps. In the last cross section shown, a complex flow pattern made up of several expansion fans and shock stems is observed, produced by the three-dimensional interactions of the forebody shock systems.

Hundreds of hours are required on conventional supercomputers such as the Cray Y-MP to perform unsteady hypersonic calculations involving nonequilibrium or reacting flow. For these calculations to be useful in the analysis and design process or to be used in a design optimization procedure, the computer time must be reduced by at least two orders of magnitude. To accomplish this, research in both numerical algorithms and computer architectures is being pursued.

\section{Elements Pacing MCAS}

Requirements for performing such simulations include multidisciplinary application software, systems software and computers powerful enough to run that software. The successful development of all three of these technologies is mandatory for a positive outcome of MCAS. There are a number of elements for MCAS that are pacing the progress not only of multidisciplinary problems but also single disciplinary problems. They include physical modeling (e.g., turbulence and transition modeling, chemistry modeling, other physics such as electromagnetics and structures), solution methodology (e.g., equation set coupling and algorithms), computer power, and multidisciplinary validation data. Secondary pacing technologies include pre- and post-data processing (e.g., surface, internal and structural definition, grid generation and scientific visualization). These pacing elements are discussed below in more detail.

\section{Solution Methodology}

With the potential for enhanced computer power offered by machines with parallel architectures will come a search for algorithms that run effectively on those machines. Not only will existing algorithms have to be programmed and evaluated, but also "old" algorithms will have to be 
revisited and tried to determine their effectiveness. In addition, the development of new algorithms specifically designed to take advantage of these architectures will have to be researched. These new algorithms should be those that can efficiently solve such equation sets as those governing gas-dynamics, structural dynamics, controls and optimization as well as some combination of those equation sets.

Because there are different parallel architectures on the market, e.g., SIMD (single instruction, multiple data) and MIMD (multiple instruction, multiple data), there will be some algorithms, e.g., explicit and implicit, that operate more effectively on each of those parallel machines. Holst et al. (ref. 1) in his paper briefly discusses some tradeoffs between algorithms and architectures. The ultimate goal, of course, is to produce the most cost-effective and accurate simulation possible and this will involve tradeoffs among numerical algorithms, computer hardware and physical disciplines.

Problems governed by multiple equation sets of different physical disciplines will require research as to the most optimum procedure for coupling those equation sets. Both indirect and direct procedures will have to be investigated. The indirect approach links the physical dependence of one discipline on another by sensitivity matrices.

The direct approach mathematically links the governing equation sets. They can be linked in either a strong or weak form. For example, hypersonic problems involve the solution of both the gas dynamic and finite-rate chemistry equations. In the strongly coupled approach, the two equation sets are solved simultaneously by a large matrix inversion process for an implicit method. In the weakly coupled approach, each equation set is solved independently at each iteration, and the information from each equation set is then used to update the other equation set for the next iteration. The strongly coupled approach takes considerably more computer time than the weakly coupled approach. For hypersonic problems the weakly coupled approach has proved successful.

In addressing problems involving multi-equation sets, the stiffness of the governing equations might also dictate the solution procedure to be employed. For example, in computing aeroelastic problems using coupled procedures, the fluid equations are cast in an Eulerian system while the structural equations are in a Lagrangian system. The structural system is much stiffer than the fluid system. Therefore, it is numerically inefficient to solve both systems using a monolithic numerical scheme. In this case a domain decomposition approach can be employed in which the equation sets are coupled only at the boundaries between the fluids and structures.
One of the advantages that computer simulation possesses over either wind tunnel or flight simulation is that it can automatically enhance the design of the aerospace vehicle or component. The tedium of design-space parameter selection and the trial-and-error process of eliminating poor designs is performed much more efficiently by the computer. This is done using numerical optimization procedures. In this approach, an optimization algorithm is coupled with a flow solver. Design requirements and constraints are imposed on the problem, and the computer is employed to search the design space until the optimal configuration is found. Research in this area is required to refine the above process.

\section{Physical Modeling}

The numerical simulation of viscous flows about rigid aerospace vehicles and their components is being attacked from both ends of the computational spectrum. At one end are Reynolds-averaged Navier-Stokes (RANS) solutions that employ suitable turbulence models while at the other end are direct numerical solutions that don't require a turbulence model. As computer and algorithmic technology improves, these two approaches will merge (ref. 5).

The development of suitable turbulence models for the RANS equations to date remains highly problem dependent. They are dependent on such elements of the problem as the free-stream conditions (for example, Mach and Reynolds number), configuration geometry (for example, deflected control surfaces and wing-body junctures) and flow field behavior (for example, shock boundary layer interaction). Accurate and numerically efficient turbulence models for numerical simulations, especially those types of models that can be used to obtain engineering answers for separated flow problems, must be developed.

One multidisciplinary area of application requiring advanced turbulence models is associated with combustion. In this area, turbulence and chemistry models must be developed to appropriately account for chemistry/ turbulence interactions. Transition models, that predict the flow characteristics leading up to fully developed turbulent flows are also important, especially for laminar flow control (LFC) applications, e.g., those associated with the high-speed civil transport, and high-speed, highaltitude flows, e.g., those associated with the National Aero-Space Plane (NASP).

To improve the turbulence-model development process, it should be considered at the early stage of code development according to Marvin (ref. 6). The first step in such a process is to identify those flows pacing the development of the aerodynamic computations. The second step is to develop models through a phased approach of 
building-block studies that combine theory, experiment and computations. The final step is to provide verification and/or limits of the modeling through benchmark experiments over a practical range of Reynolds and Mach numbers.

Turbulence models that complete the above process must then be installed in the application software. This requires close collaboration between the model developer and software author. Issues associated with overall code stability must be addressed.

\section{Computer Technology}

Conventional serial computer architectures will probably not be capable of fulfilling the requirement of multidisciplinary computational aeroscience challenges in a reasonable amount of time. For that matter, some single discipline problems, such as direct simulation of turbulence, will also demand computer power beyond that offered by serial machines. Those machines are limited by the speed of light. To alleviate that problem, computers based on parallel architectures are being developed. Massively parallel computer architectures offer another avenue for improvement in computing speed. To obtain a numerical simulation on such a computer, the problem is divided among hundreds or even thousands of processors. It is important that the work be balanced across the processors and the communication between processors is minimized. By doing this, the time to compute a solution is dramatically reduced.

The goal for the CAS element within the HPCCP is a sustained execution speed of one teraFLOPS. This will require a peak speed of more than one teraFLOPS or approximately 10 teraFLOPS. The CAS performance goal was designed to push the current state of computing technology. The HPCCP, it is hoped, will lead to the commercial development of fully scaled systems complete with system software.

Parallel computers currently available at NASA Ames for performing calculations include the Connection Machine (CM-2) with its 32,768, 7 megahertz (Mhz) processors and the Intel iPSC/860 Gamma with 128 nodes based on $40 \mathrm{Mhz}$ i860 superscalar RISC chips. The CM-2 has 128 kilobytes $(\mathrm{KB})$ of memory per processor for a total of 4 gigabytes (GB) while the Intel has $8 \mathrm{MB}$ of memory per processor for a total of IGB. Scientists at Ames also have available to them the Intel iPSC/860 Delta machine at the California Institute of Technology which has 528 processors.

These machines are currently being used by Ames scientists to solve such problems as (1) chimera grid generation for ARC3D on the CM-2, (2) implicit algorithm efficiency on the Gamma, (3) three-dimensional unstructured grids on the Gamma, (4) turbulent channel flow on the Gamma, (5) compressible homogeneous turbulence on the Gamma, (6) delta wing and highperformance aircraft wing on both the CM- 2 and Gamma, (7) strake-wing configuration for aeroelastic studies on the Gamma, (8) high-speed civil transport on the Gamma, and (9) hypersonic blunt body with chemistry using the Monte Carlo approach on the Gamma. One problem at Ames that has been quite successfully solved on a parallel machine from an efficiency point of view is a very largescale turbûlent-flow simulation.

Turbulent flow numerical simulations require extremely large data bases and long run times. To solve problems such as these on the Intel machines, Wray (ref. 7) first had to implement Vectoral (a highly optimized compiler), a very efficient FFT and an efficient inter-processor communication scheme. Once this software was written, then the applications software could be easily ported to the machines.

The first code ported simulates homogeneous turbulence in a three-dimensional box. For this code on the 128 processor Intel Gamma, it runs more than 11 times faster than on a single Cray Y-MP processor. However, that is about $25 \%$ its theoretical speed. If it were not for limitations imposed by the speed of memory accesses and of interprocessor communication, the code would run at $80 \%$ of the absolute upper bound of performance (80MFLOPS per processor in single precision). The performance on the Delta, using 512 processors, is about 30 times a Y-MP processor.

The results of these calculations indicate that highly parallel, distributed memory computers are well suited to large-scale turbulence simulations, memory speed degrades the i 860 processor performance, memory sizes for the speed available are inadequate, and the interprocessor communication speeds are out of balance with the processor speeds.

\section{Multidisciplinary Validation}

Validation of multidisciplinary application software will be a key pacing item. It takes a long time for design engineers to gain confidence in applications software. That confidence is in part gained by comparison with experimental or flight test data. It will be important to conceive and perform, in parallel with the application software development, companion experiments designed to validate the multidisciplinary computer programs being created. It is also important that these experiments be designed in concert with the MCAS scientists but at an early stage so that the data produced can impact the 
software development program in a timely fashion. In the past such attempts resulted in a missed opportunity because the experiments required such a long time to produce the validation data.

The multidisciplinary nature of the problem to be validated might preclude the validation experiment from being done in the wind tunnel because of its complicated nature. That is, the experiment could involve structures, controls and propulsion elements requiring a costly model and sophisticated instrumentation. The only alternative, therefore, would be flight testing. It's expensive, but it produces realistic data. On the other hand, simple double-disciplinary experiments can be and have been performed in the wind tunnel. A typical example is aeroelasticity in which fluid and structure interactions are studied.

A new aeroelastic experiment is being planned at NASA Ames to validate software being developed there. In it, the unsteady aerodynamic and structural responses of a flexible delta-wing configuration representative of a highspeed civil transport wing will be studied. Simple semispan models will be tested in the High-Reynolds Channel-2. Unsteady pressures will be measured using a new technology called pressure-sensitive luminescent coatings. This reduces the cost of the model. This experiment will complement aeroelastic tests being performed at NASA Langley. The data produced from this experiment will be used to validate the ENSAERO program developed by Guruswamy (ref. 8). That program couples the Euler or Navier-Stokes equations with the structural equations of motion to predict the aeroelastic response of simple wings and wing-body configurations.

\section{Secondary Pacing Technologies}

A bottleneck technology for single disciplinary, numerical fluid dynamics simulation is grid generation. The goal for grid generation, of course, is to efficiently and effectively distribute grid points to generate the most accurate solution possible with the least number of points. Research in solution adaptive procedures for steady flows has resulted in substantial gains in the optimal placement of grid points. Research is still required to effectively apply these procedures to unsteady flows which will be important in multidisciplinary computational aerosciences problems.

For multidisciplinary problems, it is quite possible that additional grid points will be required to satisfy the accuracy requirements of the secondary disciplines. Thus the grid generation process is a pacing technology for multidisciplinary problems. These problems involving multiple equation sets that describe each discipline obviously will require more time on the computer to solve. Because of this, scientists will tend to reduce the number of grid points, from that required for an accurate flow solution, in order to get their multidisciplinary problem solved in a reasonable amount of time on the computer. This impairs the overall solution accuracy and can thus bias the results. Thus, there is a need to develop solution adaptive grid procedures for unsteady problems in order to optimally utilize available grid points.

As an example, aeroelasticity problems require an unsteady flow solver to capture the physics generated by coupling the fluid and structural dynamic equations of motion. To do this for a simple wing-body configuration in a reasonable amount of time on existing computers necessitates a grid about half the size of that used for the fluids problem alone.

Numerically solved multidisciplinary problems will generate enormous amounts of data on massively parallel computers. To effectively display and digest this data, quite naturally, requires high-resolution, high-throughput computer graphics devices utilizing sophisticated software display packages. Existing packages will have to be modified to display the requisite parameters for each of the equation sets being solved.

\section{Conclusions}

Solution of the multidisciplinary computational aerosciences problems described above will go a long ways towards helping the aerospace industry fulfill its basic two objectives, that is, (1) to reduce the development cost and (2) to increase the performance, safety and environmental compatibility of aerospace vehicles and their components. The tasks are challenging but the CFD scientific community is poised to meet that challenge.

Accomplishment of those lofty goals will require computers orders of magnitude larger and faster than those currently available. It will also require systems software that permits easy use of those computers.

Massively parallel computers offer a means to meeting the multidisciplinary computational aeroscience objectives. The High-Performance Computing and Communications Program designed by NASA provides a framework and the resources necessary for systematically developing the requisite elements needed to successfully accomplish multidisciplinary simulations.

\section{Acknowledgments}

I would like to acknowledge the support provided to me in the preparation of this manuscript by Dr. Jolen Flores, 
Deputy Division Chief of the Fluid Dynamics Division at NASA Ames Research Center.

\section{References}

1. Holst, T. L.; Salas, M. D.; and Claus, R. W.: The NASA Computational Aerosciences Program Toward Teraflops Computing. AIAA-92-0558, AIAA 30th Aerospace Sciences Meeting \& Exhibit, Reno, Nevada, Jan. 6-9, 1992.

2. Smith, M. H.; Chawla, K.; and Van Dalsem, W. R.: Numerical Simulation of a Complete STOVL Aircraft in Ground Effect. AIAA Paper 91-3293-CP, AIAA 9th Applied Aerodynamics Meeting, Baltimore, Maryland, Sept. 23-25, 1991.

3. Edwards, T. A.: CFD Analysis of Hypersonic, Chemically Reacting Flow Fields. Paper No. 1, AGARD Symposium on Theoretical and
Experimental Methods in Hypersonic Flows, Torino, Italy, May 1992.

4. Lawrence, S. L.: Numerical Performance Estimates for a Generic Hypersonic Forebody. AIAA Paper 91-1695, Honolulu, Hawaii, June 1984.

5. Kutler, P.: A Perspective of Computational Fluid Dynamics. NASA TM-88246, May 1986.

6. Marvin, J. G.: Future Requirements of Wind Tunnels for Computational Fluid Dynamics Code Verifications. AIAA Paper 86-0752-CP, 1986.

7. Wray, A. A. and Rogallo, R. S.: Simulation of Turbulence on the Intel Gamma and Delta. NASA Technical Memorandum, April 1992.

8. Guruswamy, G. P.: Vortical Flow Computations on a Flexible Blended Wing-Body Configuration. AIAA Paper 91-1013, 32nd Structures, Structural Dynamics and Materials Conference, April 1991, Baltimore, Maryland. 


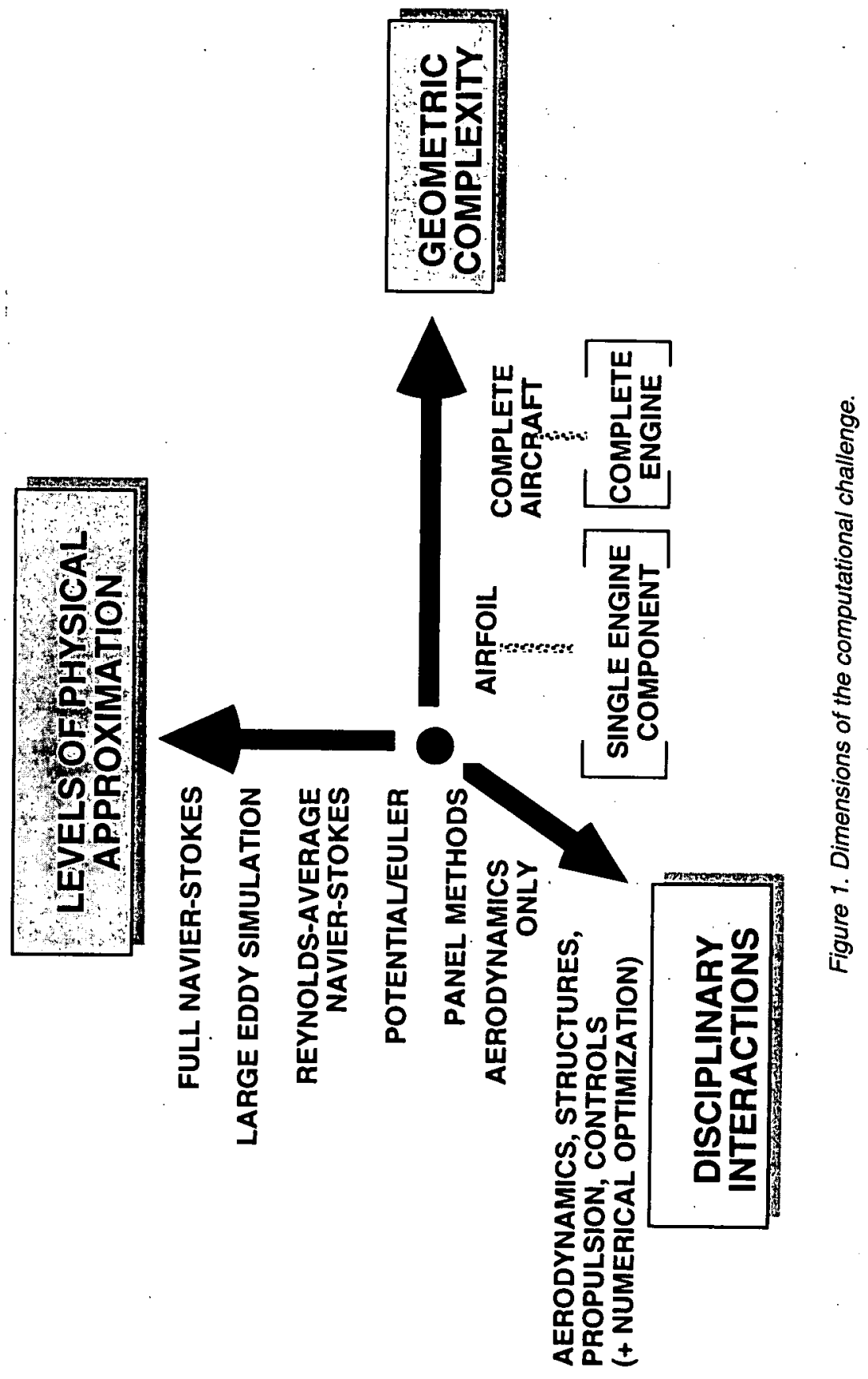




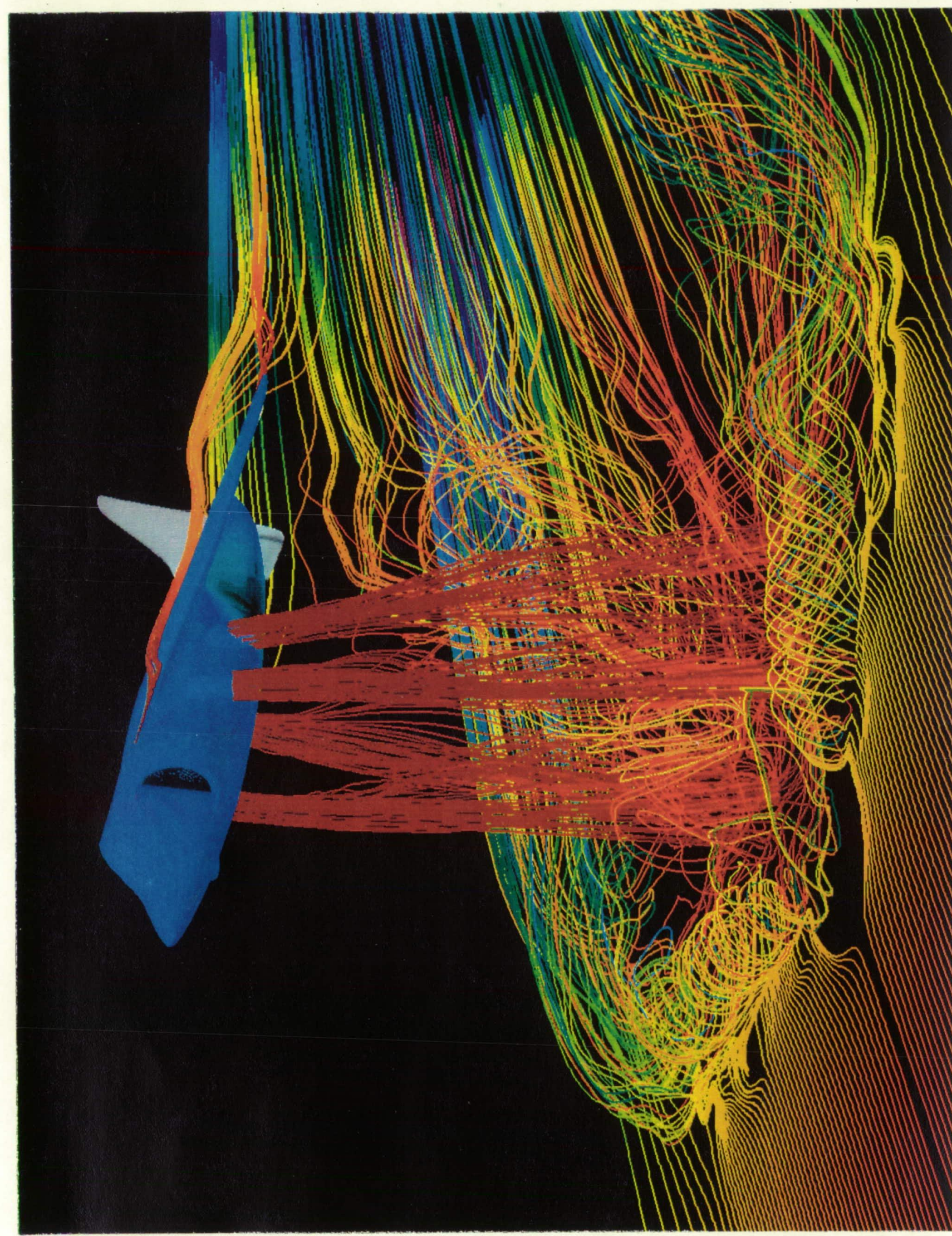




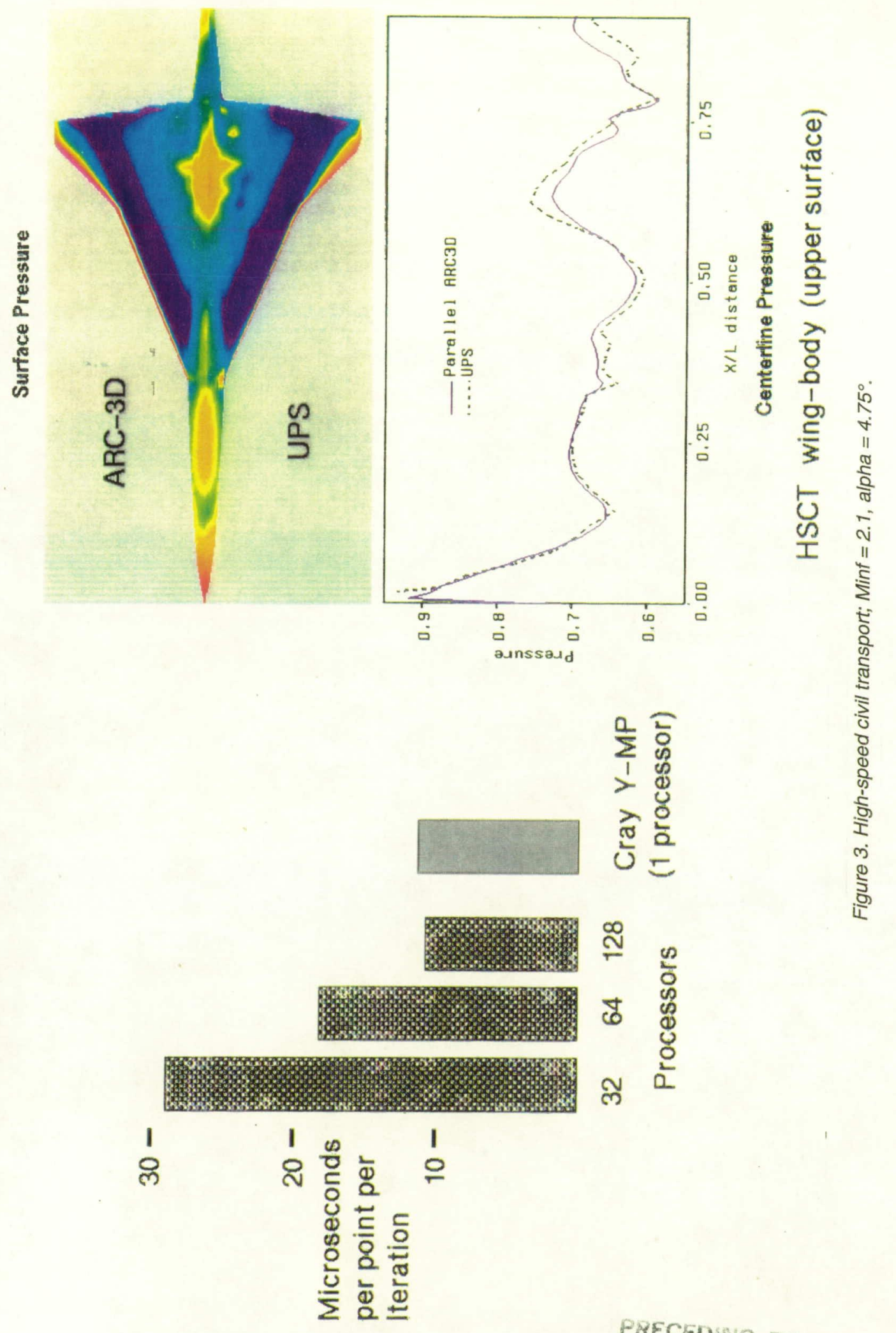

PRECEDING PAGE BLANK NOT FILMED 


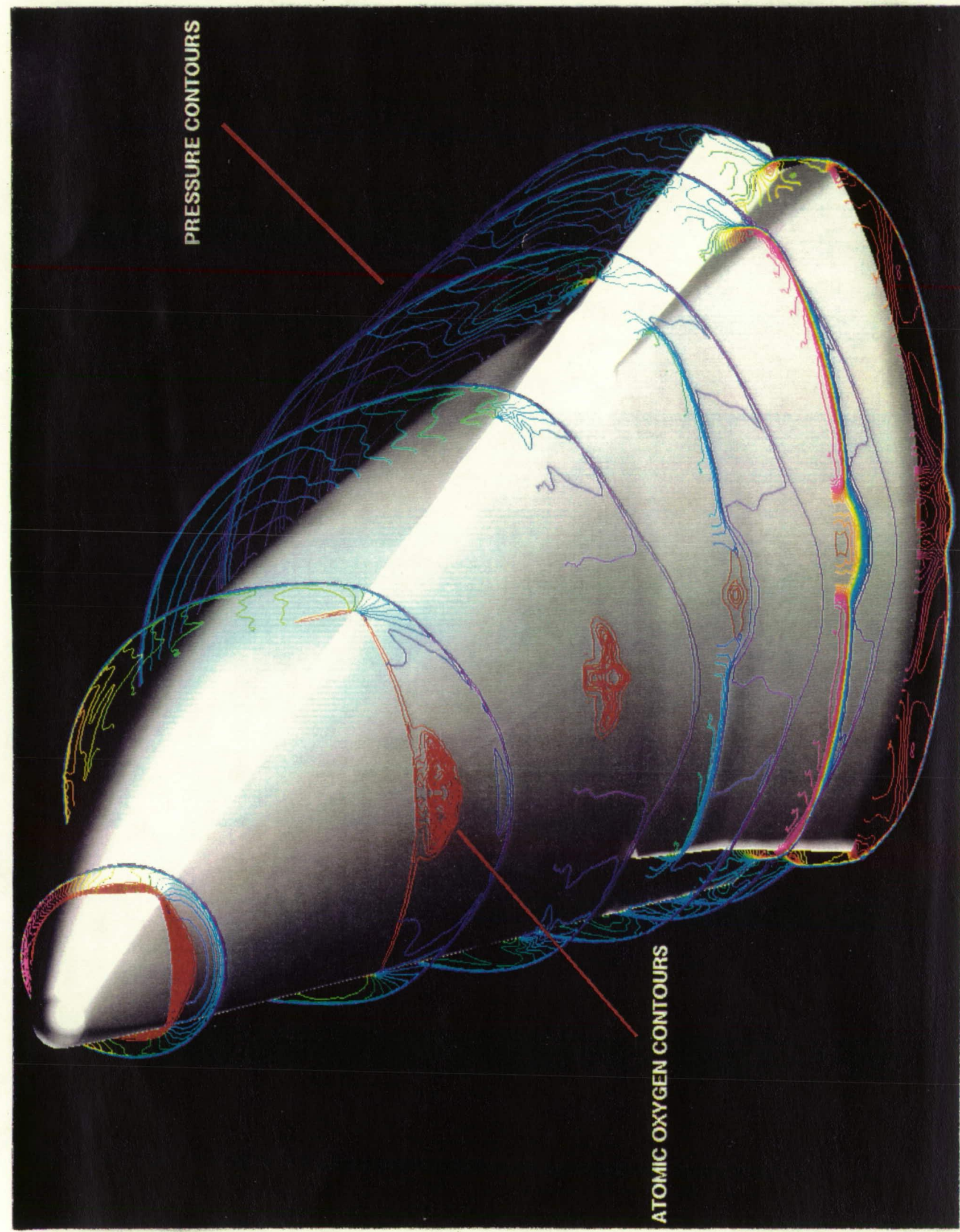

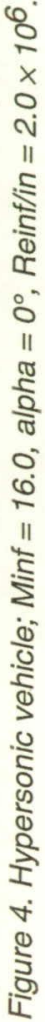

ORIGINAL PAGE

PRECEDING PAGE BLANK NOT FHLMED COLOR PHOTOGRAPH 


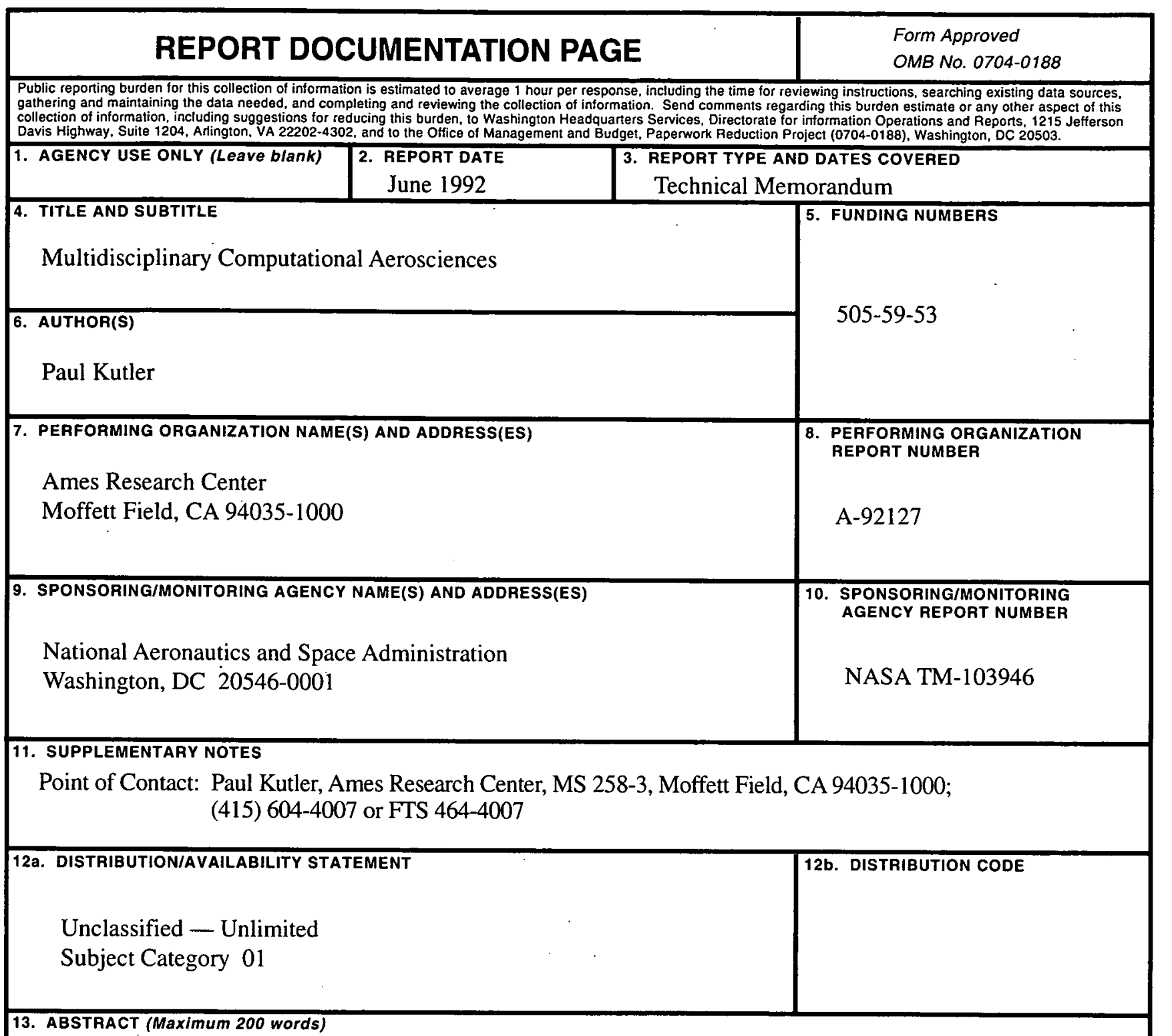

As the challenges of single disciplinary computational physics are met, such as computational fluid dynamics, computational structural mechanics, computational propulsion, computational aeroacoustics, computational electromagnetics, etc., scientists have begun investigating the combination of these single disciplines into what is being called multidisciplinary computational aerosciences (MCAS). The combination of several disciplines not only offers simulation realism but also formidable computational challenges. The solution of such problems will require computers orders of magnitude larger than those currently available. Such computer power can only be supplied by massively parallel machines because of the current speed-of-light limitation of conventional serial systems. Even with such machines, MCAS problems will require hundreds of hours for their solution. Toefficiently utilize such a machine, research is required in three areas that include parallel architectures, systems software and applications software. The main emphasis of this paper is the applications software element. Examples that demonstrate application software for multidisciplinary problems currently being solved at NASA Ames Research Center are presented. Pacing items for MCAS are discussed such as solution methodology, physical modeling, computer power and multidisciplinary validation experiments.

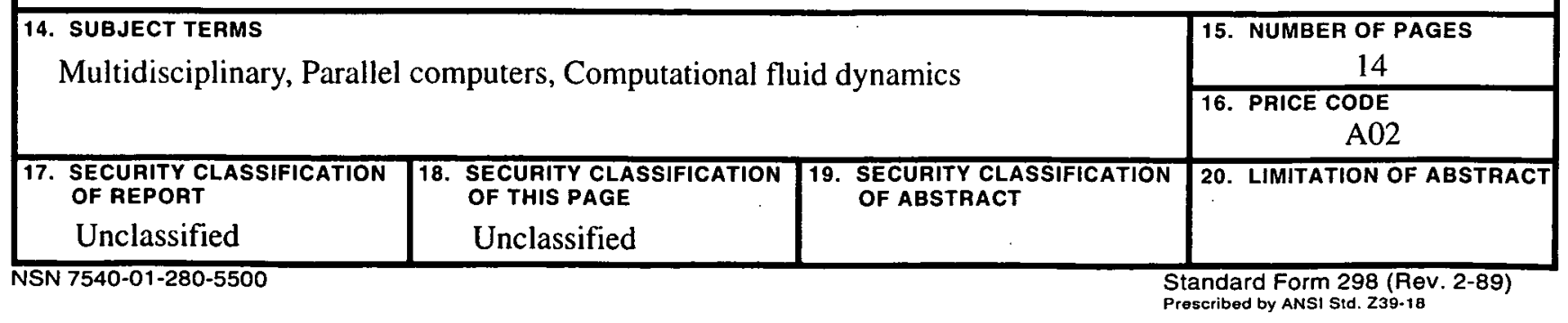

